Article

\title{
Behavioral Dynamics of Pedestrians Crossing between Two Moving Vehicles
}

\author{
Soon Ho Kim ${ }^{1}$, Jong Won Kim ${ }^{2}$, Hyun-Chae Chung ${ }^{3}$, Gyoo-Jae Choi ${ }^{4}$ and MooYoung Choi ${ }^{1, *}$ \\ 1 Department of Physics and Astronomy and Center for Theoretical Physics, Seoul National University, \\ Seoul 08826, Korea; soonhokim@snu.ac.kr \\ 2 Department of Healthcare Information Technology, Inje University, Gimhae 50834, Korea; \\ jongwonkim@inje.ac.kr \\ 3 Department of Sports and Exercise Science, Kunsan National University, Gunsan 54150, Korea; \\ hcx@kunsan.ac.kr \\ 4 School of Automotive and Mechanical Engineering, Kunsan National University, Gunsan 54150, Korea; \\ gjchoi@kunsan.ac.kr \\ * Correspondence: mychoi@snu.ac.kr; Tel.: +82-2-880-6615
}

Received: 25 December 2019; Accepted: 20 January 2020; Published: 26 January 2020

\begin{abstract}
This study examines the human behavioral dynamics of pedestrians crossing a street with vehicular traffic. To this end, an experiment was constructed in which human participants cross a road between two moving vehicles in a virtual reality setting. A mathematical model is developed in which the position is given by a simple function. The model is used to extract information on each crossing by performing root-mean-square deviation (RMSD) minimization of the function from the data. By isolating the parameter adjusted to gap features, we find that the subjects primarily changed the timing of the acceleration to adjust to changing gap conditions, rather than walking speed or duration of acceleration. Moreover, this parameter was also adjusted to the vehicle speed and vehicle type, even when the gap size and timing were not changed. The model is found to provide a description of gap affordance via a simple inequality of the fitting parameters. In addition, the model turns out to predict a constant bearing angle with the crossing point, which is also observed in the data. We thus conclude that our model provides a mathematical tool useful for modeling crossing behaviors and probing existing models. It may also provide insight into the source of traffic accidents.
\end{abstract}

Keywords: pedestrian behavior; human locomotion; human dynamics; traffic safety

\section{Introduction}

Pedestrians make up a large portion of traffic accident fatalities, particularly in areas of high population density [1,2]. Exploring the behavioral dynamics of road crossing may provide insight into the fundamental source of accidents [2-4]. The task of crossing a road involves a goal-directed movement, as the pedestrian desires to reach the other side of the street subject to the avoidance condition due to passing vehicles. This relates the problem to studies on human behavior during tasks of avoidance $[3,5]$ as well as interception [6-8].

The constant bearing-angle model has gained attention as a possible strategy that humans employ in order to intercept moving objects [6-8]. This walking strategy is tied to how human locomotion is visually controlled [9-12]. In addition, studies involving movements through gaps have employed the concept of affordance, the possibilities for action constrained by the environment and physical conditions of the actor [13-15]. Statistical analyses of pedestrian inter-vehicle gap acceptance rates, which depend on the pedestrian's perception of affordance, have also been reported [16]; these studies, however, do not typically provide a dynamic model of action. 
The purpose of this study is to develop a model of road crossing that can be used to analyze data and test hypotheses. Specifically, we examine how pedestrians cross a street between two moving vehicles. An experiment is constructed in which human participants cross a street within a virtual environment with a range of experimental conditions. A mathematical model is developed which accurately describes typical crossing patterns. The model is applied to experimental data via minimization of the root-mean-square deviation (RMSD) with respect to the model parameters. When the best fit parameters are found, the model equations give us the position of the pedestrian as a function of time.

Discussing the meaning of each parameter of the model, we examine how the average value of each parameter varies depending on crossing conditions. Specifically, we consider the effects of adjusting the gap size and the initial distance as well as the pedestrian's age, vehicle speed, and vehicle type. We further make use of our model to derive an inequality among the parameters that must be satisfied for successful crossing to occur, and, accordingly, to describe the affordance $[13,15]$ of the crossing situation. The possibility of intercepting the gap between the cars has been mathematically modeled with the environmental factors (e.g., speed of cars, length of lanes) and the walker's capabilities such as walking speed and response time. It is also observed in the data that the bearing angle tends to be constant with respect to the crossing point, which is obtained analytically from the model equations.

In Section 2, we describe the experimental procedure and lay out our model. The concept of affordance and bearing angle are also formulated using the model. In Section 3, we draw conclusions from the fitting parameters of the model. The results are visualized and analyzed in the affordance and bearing-angle viewpoints. In Section 4, we discuss the implications of our results and we conclude in Section 5 .

\section{Methods}

\subsection{Data Collection}

Sixteen children (of age $12.2 \pm 0.8$ yrs, i.e., mean age 12.2 years and standard deviation 0.8 years), sixteen young adults (of age $22.8 \pm 2.6 \mathrm{yrs}$ ), and fourteen elderly people (of age $54.1 \pm 4.9 \mathrm{yrs}$ ) with normal or corrected-to-normal vision were recruited for this experiment. Informed written consent was obtained from all individual participants. The experiment was conducted in accordance with the Declaration of Helsinki, and the protocol was approved by the Kunsan National University Research Board. Each subject was placed on a customized treadmill (of dimensions $0.67 \mathrm{~m}$ wide, $1.26 \mathrm{~m}$ long, and $1.10 \mathrm{~m}$ high) with four magnetic counters that track movements. A Velcro belt connected to the treadmill is worn to decrease vertical and lateral movements, and a handrail is placed for stability. The treadmill turns with minimal friction as the participants walk, and magnetic counters track rotations (left panel, Figure 1). Each participant wore an Oculus Rift (Menlo Park, CA, USA) DK1 virtual reality headset connected to a standard desktop PC. The headset portrayed a realistic view of a street with a crosswalk in $1280 \times 800$ resolution 3D stereoscopic visual images that respond realistically according to the participant's movements. Practice trials were performed prior to the experiments to familiarize each participant with the treadmill and virtual environment before the experiment. (see [4] for details).

A diagram of the virtual crossing situation is given in Figure 2. The participant, standing at rest before a car lane with a "ready" signal shown, was instructed to press a button when a "go" signal appears, and then to look at her/his left side, from which two vehicles, one in front of the other, were moving at equal constant speed $v_{c}$. The participant was instructed to cross between the two vehicles if she/he believed she/he could cross successfully. If a collision with a vehicle occurred, the simulations halted. The experimenter recorded whether there was a successful crossing, a collision, or no crossing. 


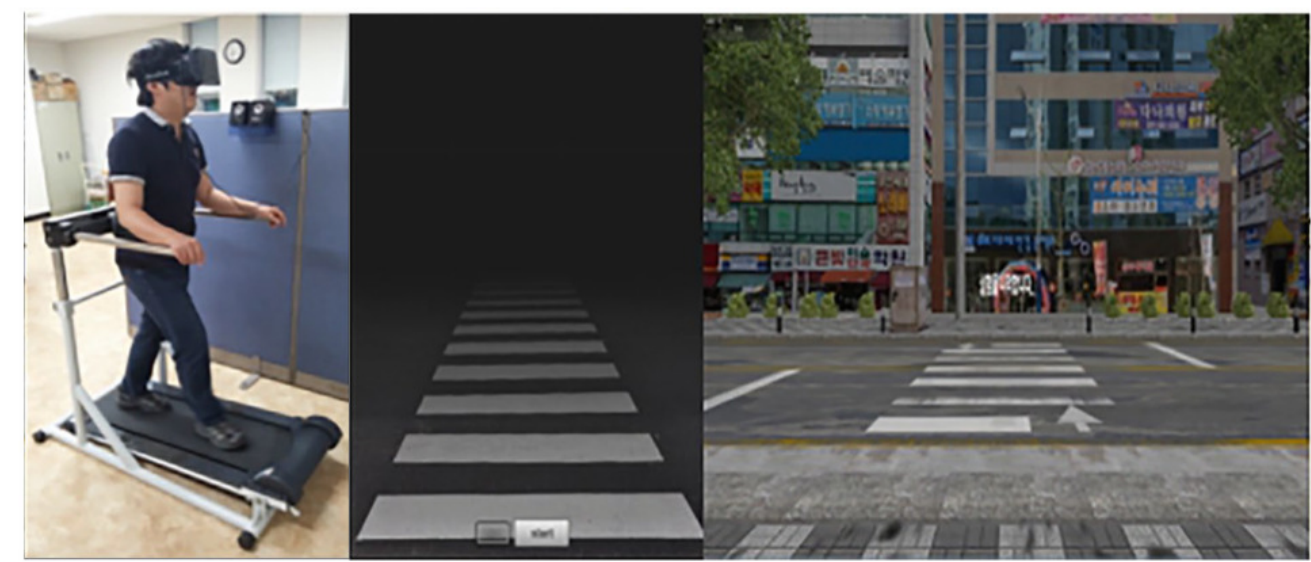

Figure 1. Images of experimental setup. Left panel: photograph of student participating in an experiment. Middle panel: cartoon crosswalk used for calibration. Right panel: Screenshot of the virtual crosswalk used in the experiment.

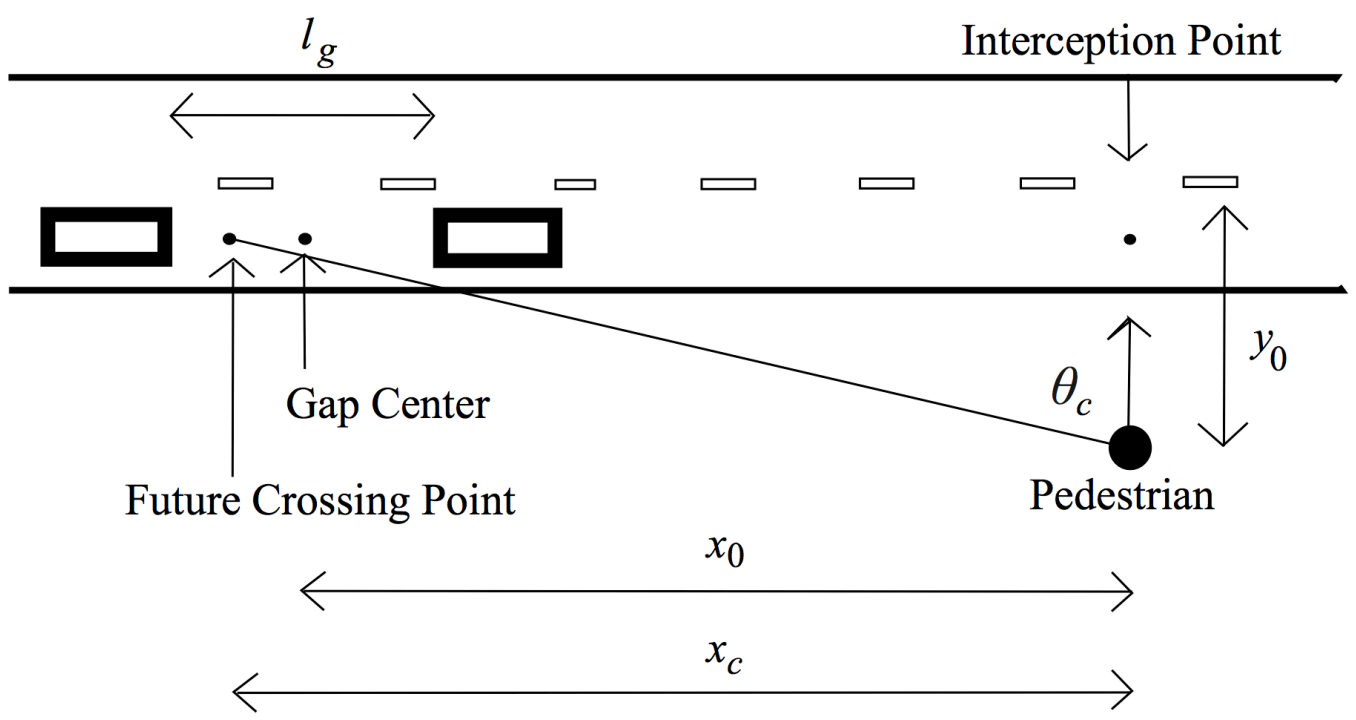

Figure 2. Top-down diagram of the crossing environment at time $t=0$. The two boxes on the road depict two vehicles facing the right, which move forward at a constant speed. The circle depicts the pedestrian, who begins at rest and is facing in the direction the arrow. Labeled are experimental parameters $l_{g}, y_{0}$, and the bearing angle to the crossing point $\theta_{c}$.

The experiment was designed to examine the participants' responses to a single gap, and so only two vehicles were present. We examine the responses of the participants to changing gap characteristics by varying experimental parameters. These include pedestrian starting position $y_{0}$, gap time $t_{g}$ (or gap length $l_{g}$ ), vehicle speed $v_{c}$, and the vehicle type (sedan or bus). Details of age groups and a full list of experimental parameters are given in Table 1.

The gap center is defined to be the midpoint of the gap between the vehicles. While the width of the gap and speed of the vehicles are varied, the initial position $x_{0}$ of the gap center is always set in such a way that the gap center reaches the interception point at time $t=4 \mathrm{~s}$. Accordingly, we have $x_{0}=-v_{c} \times 4 \mathrm{~s}$. 
Table 1. Table summarizing age groups and experimental parameters.

\begin{tabular}{cc}
\hline Participant Group & Number of Participant \\
\hline Children & 16 \\
\hline Young adults & 16 \\
\hline Elderly & 14 \\
\hline Experimental Parameter & Values Used \\
\hline$y_{0}$ (initial distance) & $-3.5,-4.5,-5.5,-6.5 \mathrm{~m}$ \\
\hline$t_{g}$ (gap time) & $2.5,3,4 \mathrm{~s}$ \\
\hline$v_{c}$ (vehicle speed) & $30,60 \mathrm{~km} / \mathrm{h}$ \\
\hline vehicle type & sedan, bus \\
\hline total configurations & 48 \\
\hline
\end{tabular}

\subsection{Model}

The following model, called the simple crossing model, was used to analyze crossing data. The velocity is assumed to follow a logistic function of time in the form

$$
v(t)=v_{\max } \frac{\exp \left[\left(t-t_{a}\right) / \tau\right]}{1+\exp \left[\left(t-t_{a}\right) / \tau\right]},
$$

which, upon integration, results in the position as a function of time:

$$
y(t)=y_{0}+v_{\max } \tau \log \left\{1+\exp \left[\left(t-t_{a}\right) / \tau\right]\right\} .
$$

Equation (2) is plotted in Figure $3 a$ (red line). Constants $t_{a}, \tau, v_{\max }$ are fitting parameters whose meanings can be understood as follows: The measurement begins at time $t=0$. Assuming $t_{a}-2 \tau>0$, we have the initial position and velocity of the pedestrian, $y(t=0) \approx y_{0}(<0)$ and $v(t=0) \approx 0$, respectively. Then, the pedestrian accelerates smoothly until the maximum velocity $v_{\max }$ is reached. The parameter $\tau$ then serves as a measure for the duration of this acceleration, the midpoint between which is given by $t_{a}$. Note that, at time $t=t_{a}-2 \tau$, the velocity in Equation (1) becomes $v(t=$ $\left.t_{a}-2 \tau\right)=v_{\max } e^{-2} /\left(1+e^{-2}\right) \approx 0.1 v_{\max }$. While Equation (1) never gives $v=0$ exactly, in practice, we may define $t_{d} \equiv t_{a}-2 \tau$ to be the time at which the pedestrian begins to accelerate forward. If preferable, one may take alternatively $t_{d} \equiv t_{a}-3 \tau$, which corresponds to $v\left(t=t_{d}\right) \approx 0.01 v_{\max }$.

A second model, called the two-step crossing model, is used to analyze crossings that have more than one acceleration event and thus do not fit the simple crossing model (Figure $3 \mathrm{~b}$ ). The two-step crossing model is discussed in the Appendix A.

Each piece of data classified as a simple crossing is fit to Equation (2) by minimizing RMSD with respect to the fitting parameters. We probe the effects of gap characteristics by examining how the distributions of parameters change with the variation of certain features of the gap, and discuss the results in Section 3.2. Those data displaying the two-step pattern are fitted separately to the extended model, and the results are discussed in the Appendix A.

\subsection{Affordance}

Affordance stands for the range of possible actions that the environment offers to the acting agent. In the crossing task, the affordance is determined by how long the gap overlaps with the participant's walking trajectory. Assuming the simple crossing model (i.e., Equations (1) and 2)), the affordance of the gap is described by the inequality 


$$
\begin{aligned}
& t_{f}-\tau \log \left[e^{\left(-y_{0}-w / 2\right) / v_{\max } \tau}-1\right]<t_{a} \\
& \quad<t_{b}-\tau \log \left[e^{\left(-y_{0}+w / 2\right) / v_{\max } \tau}-1\right] .
\end{aligned}
$$

Here, $t_{f} \equiv\left|x_{0}+l_{g} / 2\right| v_{c}^{-1}$ corresponds to the time at which the back bumper of the leading vehicle passes the intersection point and $t_{b} \equiv\left|x_{0}-l_{g} / 2\right| v_{c}^{-1}$ corresponds to the time at which the front bumper of the trailing vehicle passes the point, while $w$ denotes the width of the vehicles and equals $1.5 \mathrm{~m}$ in our experiment. $t_{f}$ is hence manifested in Figure $3 \mathrm{a}$ by the time coordinate of the right side of the box to the left $(2.5 \mathrm{~s})$, while $t_{b}$ is by that of the left side of the box to the right (5.5 s). Equation (3) thus describes the condition under which the pedestrian's trajectory passes between the two boxes in Figure 3a.

(a)

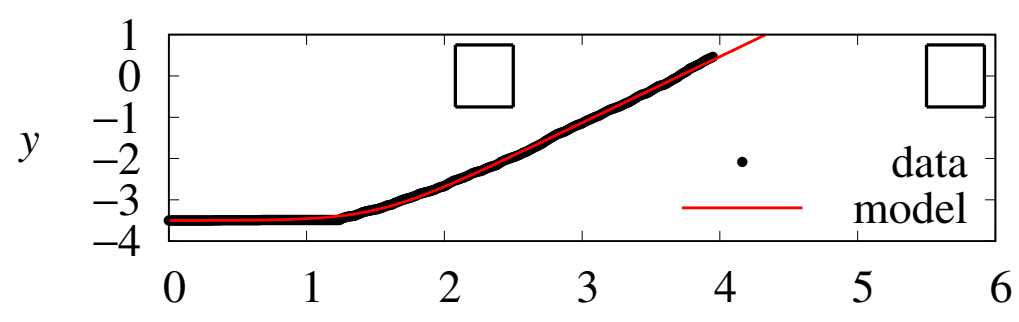

(b)

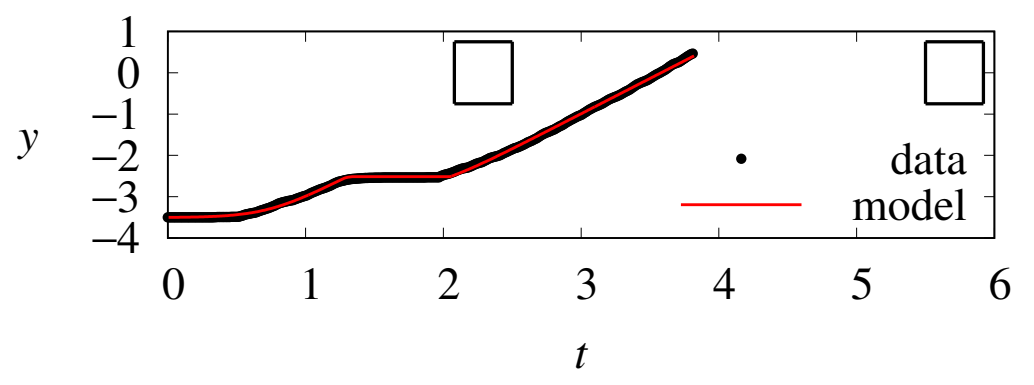

Figure 3. Pedestrian position-time plots illustrating typical crossing patterns. Black traces indicate example data; red traces indicate the corresponding model fits. Left and right boxes indicate the temporal and spatial area that the leading and the trailing vehicles occupy, respectively. Intersecting one of the box lines would indicate a collision. In both examples, the conditions are described by $y_{0}=-3.5 \mathrm{~m}, t_{g}=3.0 \mathrm{~s}$, and $v_{c}=30 \mathrm{~km} / \mathrm{h}$, with the vehicle type set to be a sedan. (a) an example of the simple cross with a single acceleration event followed by constant speed walking; (b) an example of the two-step cross with two acceleration events.

In general typical values of $\tau$ are small than the time scale of crossing, e.g., compared with $\left(-y_{0} \pm w / 2\right) / v_{\max }$. (Note that $y_{0}<0$ in our coordinate system.) Accordingly, we may take the limit $\tau \rightarrow 0$, and reduce Equation (3) to

$$
t_{f}-\frac{1}{v_{\max }}\left(-y_{0}-\frac{w}{2}\right)<t_{a}<t_{b}-\frac{1}{v_{\max }}\left(-y_{0}+\frac{w}{2}\right) .
$$

This provides a simpler inequality involving two fitting parameters. 


\subsection{Bearing Angle}

Dynamics of interceptive movement are often described in terms of the bearing angle $[7,8]$, which refers to the angle between the velocity vector of the human subject and the line of sight between the subject and the object she/he hopes to intercept. In brief, this model asserts that people intercept a moving object by choosing such a trajectory that the bearing angle is kept constant.

Our case of crossing a road may be cast into an interception task: The pedestrian must "intercept" the empty gap between the vehicles [17]. We may hence apply the bearing angle approach to our crossing experiment and model. One difficulty with this approach is that the gap is not a point but a moving area. As an obvious choice, we may simply use the gap center $x_{g}(t)$, with respect to which the bearing angle is $\theta_{g}(t)=\arctan \left[x_{g}(t) / y(t)\right]$. However, the pedestrian may not cross the gap center; it is thus more appropriate to examine the bearing angle with respect to the point within the moving gap that the pedestrian actually crosses. With $t^{*}$ denoting the crossing time, we have $y\left(t^{*}\right)=0$ and let the position of the gap center at the crossing time be $\Delta x$, i.e., $x_{g}\left(t^{*}\right)=\Delta x$. We then define the crossing point,

$$
x_{\mathcal{c}}(t)=x_{g}(t)-\Delta x=x_{0}-\Delta x+v_{\mathcal{c}} t=v_{\mathcal{c}}\left(t-t^{*}\right),
$$

and consider the angle with respect to $x_{c}$ :

$$
\theta_{c}(t)=\arctan \left[\frac{x_{c}(t)}{y(t)}\right]
$$

Taking the time derivative of Equation (6) results in

$$
\dot{\theta_{c}}=\frac{x_{c} y}{x_{c}^{2}+y^{2}}\left(\frac{\dot{x}_{c}}{x_{c}}-\frac{\dot{y}}{y}\right)
$$

Assuming that $y$ follows the simple crossing model (i.e., Equation (2)), $|\dot{y}|$ is small when $t<t_{a}$. Considering the signs of variables (especially, $x_{c}<0$ and $\dot{x}_{c}>0$ ), we thus have that $\dot{\theta}_{c}<0$, indicating a decreasing bearing angle. When $t>t_{a}+2 \tau$, the speed approaches the maximum: $\dot{y} \approx v_{\max }$, so that we have

$$
\frac{\dot{x}_{c}}{x_{c}}-\frac{\dot{y}}{y} \approx \frac{v_{c}}{v_{\mathcal{c}}\left(t-t^{*}\right)}-\frac{v_{\max }}{v_{\max }\left(t-t^{*}\right)}=0,
$$

which, upon substituting into Equation (7), yields $\dot{\theta}_{c}=0$ or a constant bearing angle. The model thus predicts that the bearing angle should decrease at the first stage of crossing and remain constant thereafter. The constant value $\theta_{c}^{*}$ that the bearing angle approaches can be estimated by

$$
\begin{aligned}
\lim _{\Delta t \rightarrow 0} \theta_{\mathcal{c}}\left(t^{*}-\Delta t\right) & =\lim _{\Delta t \rightarrow 0} \arctan \left(\frac{v_{c} \Delta t}{v_{\max } \Delta t}\right) \\
& =\arctan \left(\frac{v_{\mathcal{c}}}{v_{\max }}\right) .
\end{aligned}
$$

\section{Results}

\subsection{Data Analysis}

Tables 2 and 3 show the percentage of successful crossings in this group and the proportions of two-step crossings to the total successful crossings. The success rate drops significantly when the gap length is made small at $20.8 \mathrm{~m}$ but still stays above $80 \%$. The highest proportion of two-step crossings occurs when the gap is the shortest and the walking distance is the furthest. 
Table 2. Proportion of successful crossings to all crossing attempts for several values of $y_{0}$ and $l_{g}$, when $v_{\mathcal{C}}=30 \mathrm{~km} / \mathrm{h}$ and vehicle type is sedan.

\begin{tabular}{|c|c|c|c|}
\hline \multicolumn{4}{|c|}{ Successful Crossings } \\
\hline$y_{0}(\mathrm{~m}) \quad l_{g}(\mathrm{~m})$ & 20.8 & 25 & 33.3 \\
\hline-3.5 & $88 \%$ & $100 \%$ & $100 \%$ \\
\hline-4.5 & $100 \%$ & $100 \%$ & $100 \%$ \\
\hline-5.5 & $100 \%$ & $94 \%$ & $100 \%$ \\
\hline-6.5 & $82 \%$ & $94 \%$ & $100 \%$ \\
\hline
\end{tabular}

Table 3. Proportion of two-step crossings to all successful crossings for several values of $y_{0}$ and $l_{g}$, when $v_{c}=30 \mathrm{~km} / \mathrm{h}$ and vehicle type is sedan.

\begin{tabular}{|c|c|c|c|}
\hline \multicolumn{4}{|c|}{ Two-Step Crossings } \\
\hline$y_{0}(\mathrm{~m}) \quad l_{g}(\mathrm{~m})$ & 20.8 & 25 & 33.3 \\
\hline-3.5 & $42 \%$ & $25 \%$ & $6 \%$ \\
\hline-4.5 & $31 \%$ & $0 \%$ & $0 \%$ \\
\hline-5.5 & $0 \%$ & $26 \%$ & $0 \%$ \\
\hline-6.5 & $15 \%$ & $6 \%$ & $0 \%$ \\
\hline
\end{tabular}

Equation (2) was fit to simple crossings with an average RMSD of $0.068 \mathrm{~m}$. The low RMSD values indicate that the model accurately describes the majority of crossings. Two-step crossings were also found to be accurate, and are discussed in the Appendix A. Examples of the model equations fit to simple and two-step crossing time series are given in Figure 3a,b, respectively.

\subsection{Behavioral Response to Gap Features}

Restricting the analysis to simple crossings, we consider the variations of the parameters to changing crossing conditions. Experimental parameters $y_{0}$ and $l_{g}$ affect directly the affordance of the gap by changing the temporal window of the gap or the distance the pedestrian needs to traverse to reach the gap. Effects of the experimental parameters on the three fitting parameters $v_{\max }, t_{a}$, and $\tau$ have been examined; only $t_{a}$ has turned out to respond significantly. Figure 4 shows the distribution of $t_{a}$ obtained for several values of $y_{0}$ and $l_{g}$. It is observed that $t_{a}$ generally increases as $y_{0}$ approaches zero. This can be understood intuitively as follows: Recall that $y_{0}$ denotes the distance the pedestrian must traverse to reach the gap. The larger the distance, the earlier they must begin walking. However, when the initial position is farther, namely, when $y_{0}$ is made larger, this trend disappears and $t_{a}$ tends to stay at slightly over one second $\left(t_{a} \gtrsim 1 \mathrm{~s}\right)$. This is likely to result from the minimum response time. Namely, the pedestrian may not cross earlier than the earliest timing at which they can reasonably begin to walk. On the other hand, an increase in the gap size appears to lower $t_{a}$. This indicates that, when the gap is accessible earlier, the pedestrian tends to cross earlier. The distributions of the other two parameters $v_{\max }$ and $\tau$ have also been examined. While the average value of $v_{\max }$ tends generally to increase with $y_{0}$, the trend is not statistically significant. No significant trends have been observed for $\tau$.

Contrary to $y_{0}$ and $l_{g}$, the vehicle speed $v_{c}$ and the vehicle type are manipulated without changing the gap affordance. These experimental parameters affect the visual perception of the gap without changing its temporal window of availability. Figure 5 displays the effects of the vehicle speed and type on $t_{a}$ when the gap time $t_{g}$ is set to be $3 \mathrm{~s}$ and $y_{0}$ to be $-3.5 \mathrm{~m}$. Doubling the vehicle speed results 
in a significant increase in $t_{a}$. Moreover, in several cases, buses resulted in a greater value of $t_{a}$ than sedans did.

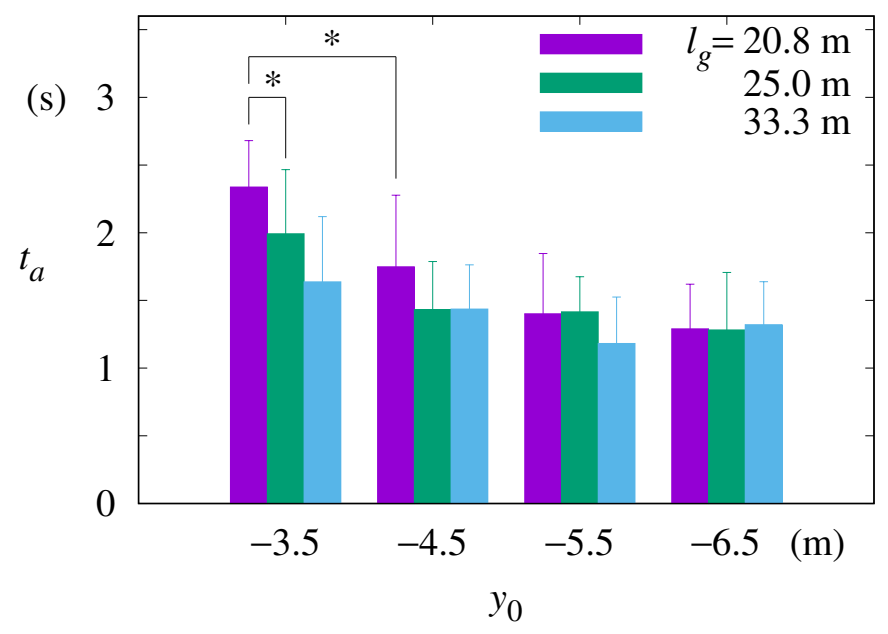

Figure 4. Distributions of parameter $t_{a}$ for varying $y_{0}$ and $l_{g}$. Here, $v_{\mathcal{c}}=30 \mathrm{~km} / \mathrm{h}$ and vehicle type is sedan. Columns indicate the average values of $t_{a}$ in the data for given experimental conditions while error bars represent standard deviations. Pairs of samples, marked with asterisks, are presumed to belong to different distributions $(p<0.05)$ according to the Mann-Whitney $U$ test. (Note here that not all such pairs are marked.)

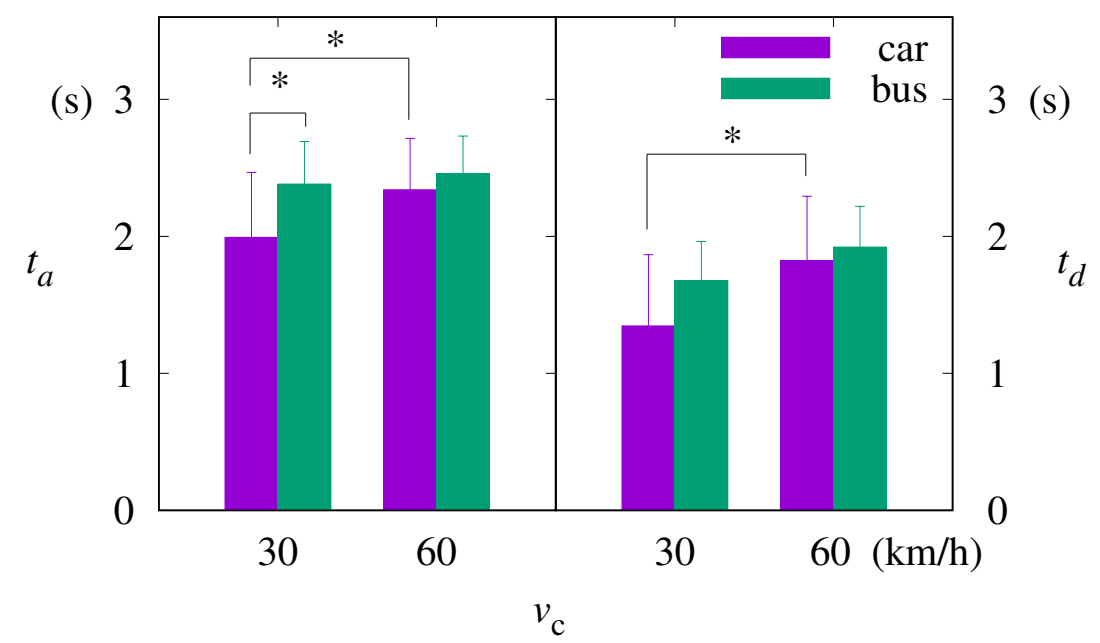

Figure 5. Distributions of parameters $t_{a}$ (left) and $t_{d}$ (right) for varying vehicle speeds $v_{c}$ and for two vehicle types. Other parameters are set to $t_{g}=3 \mathrm{~s}$ and $y_{0}=-3.5 \mathrm{~m}$. Columns indicate the average values in the data for given experimental conditions while error bars represent standard deviations. Pairs of samples, marked with asterisks, are presumed to belong to different distributions $(p<0.05)$ according to the Mann-Whitney $U$ test.

On the other hand, when the same comparison is made for data with $y_{0}<-3.5 \mathrm{~m}$, there arises no significant shift in $t_{a}$ or $t_{d}$ upon changing the vehicle type. For $y_{0}<-4.5 \mathrm{~m}$, no significant shift is observed upon changing the vehicle speed as well. This suggests that, when the initial distance is sufficiently far, pedestrian's judgement of the gap is hardly affected by the vehicle type or speed.

Finally, we examine differences among age groups. According to the Mann-Whitney $U$ test, the difference in the distribution of $v_{\max }$ is found to be significant $(p<0.05)$ when either the young adult group or the elderly group is compared with the child group. Both the young adult and elderly groups consistently have higher average values of $v_{\max }$ across all crossing conditions, by about $0.3 \mathrm{~m} / \mathrm{s}$. 
While children have generally slightly lower values of $t_{a}$, perhaps a sign of earlier start up times to compensate for their lower speeds, the differences are not found to be statistically significant. The young adult and elderly groups do not show significant differences.

\subsection{Parameters Fall within Affordance}

Due to the accuracy of the simple crossing model, we expect Equations (3) and (4) to hold for the fitting parameters derived from the data. Figure 6a presents the affordance boundaries in the 3D parameter space. The curved surfaces depict the boundaries specified by Equation (3), and each data point plots the parameters corresponding to a single crossing. For comparison, data for two-step crossings (crosses) as well as simple crossings (circles) are displayed. It is observed that the circles, corresponding to simple crossings indeed lie within the volume between the boundaries. In contrast, most of the crosses are located outside, above the higher surface. This indicates that the subject is on route to collide with the leading vehicle and therefore deceleration is necessary.

(a)

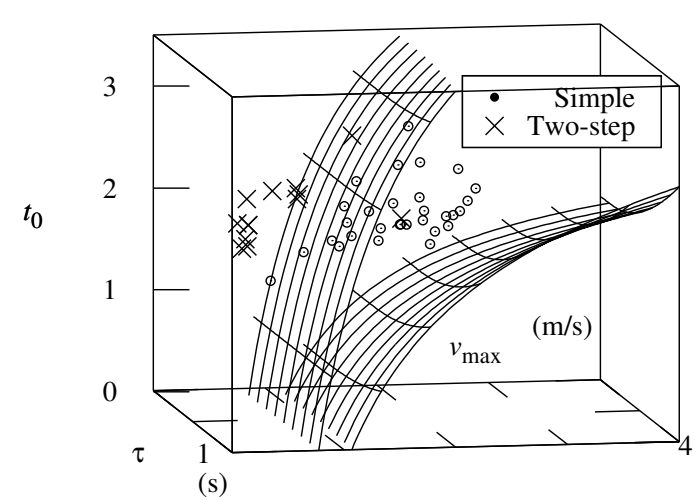

(b)

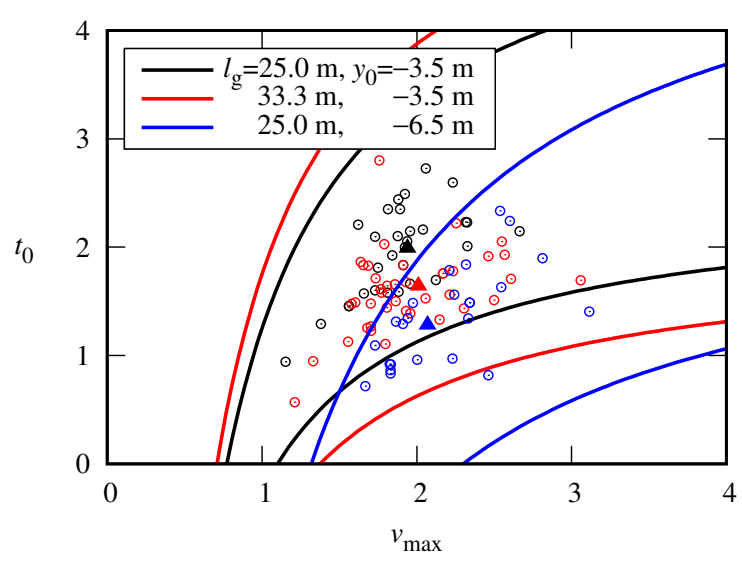

Figure 6. Data plotted with boundaries representing the affordance of the gap. (a) data plotted with surfaces in the three-dimensional parameter space $\left(\tau, v_{\max }, t_{a}\right)$ defined by Equation (3). Data points for $l_{g}=25 \mathrm{~m}$ and $y_{0}=-3.5 \mathrm{~m}$ are plotted for all age groups; dots represent simple crossings and crosses represent two-step crossings; (b) data plotted with boundaries on the two-dimensional plane $\left(v_{\max }, t_{a}\right)$ defined by Equation (4). To illustrate the effects of a shift in affordance, data and boundaries for $l_{g}=25 \mathrm{~m}$ and $y_{0}=-3.5 \mathrm{~m}$ (black) are plotted with those for $l_{g}$ changed to $33.3 \mathrm{~m}$ and for $y_{0}$ changed to $-6.5 \mathrm{~m}$ are shown in red and in blue, respectively. Triangles indicate average parameter values. 
Plotting Equation (4) on the 2D parameter plane $\left(v_{\max }, t_{a}\right)$ corresponds to the projection of the 3D plot in Figure 6a onto the plane defined by $\tau=0$. This results in Figure $6 \mathrm{~b}$, where two more cases have been included in addition to the case of the gap length $l_{g}=25 \mathrm{~m}$ and the initial position $y_{0}=-3.5 \mathrm{~m}$ presented in Figure 6a. Namely, to probe how the distribution of parameter values shifts with $l_{g}$ and $y_{0}$, we consider the data for a larger gap $l_{g}=33.3 \mathrm{~m}$ and for a farther initial position $y_{0}=-6.5 \mathrm{~m}$. Accordingly, Figure $6 \mathrm{~b}$ presents data for three sets of the gap length and initial position together with the corresponding boundaries (lines instead of surfaces in Figure 6a) determined by Equation (4). Specifically, the cases of $\left(l_{g}=33.3 \mathrm{~m}, y_{0}=-3.5 \mathrm{~m}\right)$ and $\left(l_{g}=25 \mathrm{~m}, y_{0}=-6.5 \mathrm{~m}\right)$ are plotted in red and in blue, respectively, as well as the case of $\left(l_{g}=25 \mathrm{~m}, y_{0}=-3.5 \mathrm{~m}\right)$ plotted in black. It is observed that, as the gap is widened, the average behavior (designated by red triangles) shifts toward smaller $t_{a}$ and larger $v_{\max }$. This reflects the tendency of the pedestrian to cross early before the gap center when possible. On the other hand, in the case that the initial position becomes farther from the intersection point, the pedestrian must compensate by either beginning to walk earlier or walking faster. Data in blue indeed exhibit on average a decrease in $t_{a}$ and a slight increase in $v_{\max }$ (which is, however, not statistically significant). We remark that Figure $6 \mathrm{~b}$ corresponds directly to Figures $3 \mathrm{C}$ and 3D of [13] while Figure 6a is a generalization.

\subsection{Bearing Angle Analysis}

Finally, we examine the bearing angle of the data and compare it with the model predictions. Figure 7 shows the bearing angle as a function of time for two sets of data (colored lines). The bearing angle tends to decrease in the first few seconds of crossing and to remain constant thereafter, as predicted by Equation (9). In addition, the analytical results given by Equation (6) are plotted with the average parameter values (black line). Both the theory (analytical result from the model) and the experiment (result computed from data) show that a constant bearing angle is held once the pedestrian starts moving at a nearly constant speed. It is shown in Figure 7 that the time interval during which the constant angle is observed is significantly shorter for $y_{0}=-3.5 \mathrm{~m}$ (red) than for $y_{0}=-6.5 \mathrm{~m}$ (cyan). This reflects the smaller value of $t_{a}$ in the latter case, when the initial position is farther from the interception point.

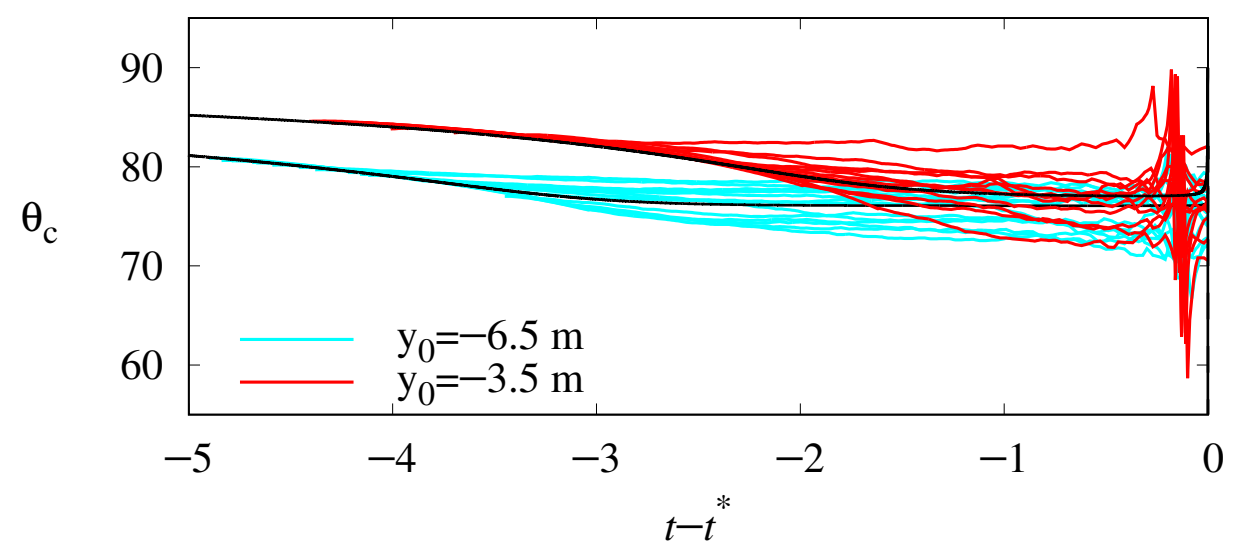

Figure 7. Time evolution of the bearing angle $\theta_{\mathcal{c}}$, defined by Equation (6). The gap length is $l_{g}=25 \mathrm{~m}$ and the initial position is $y_{0}=-3.5 \mathrm{~m}$ and $-6.5 \mathrm{~m}$ for red and cyan lines, respectively. Colored lines are computed from data, while black lines show the analytical results using average parameter values. The time axis is given in terms of time before crossing $t-t^{*}$, so that the zero point is equal to the intersection time of the run (i.e., the time at which $y(t)=0$ ).

We note that the fluctuations of the data in Figure 7 are due to measurement error, which is magnified immediately before the pedestrian meets the crossing point. This can be seen in Equation (5) 
by considering that $y(t) \rightarrow 0$ as $t \rightarrow t^{*}$, causing the error in the argument of the arctan function to become magnified.

\section{Discussion}

In this study, we have proposed a model for pedestrian crossing and utilized it to extract information from experimental data. The model fit the data with high accuracy, allowing for applications of different methods. In particular, the model allows us to visualize the affordance of each gap and see whether the data lies within it. The model also predicts a constant bearing angle, which has been observed in the data.

The fitting parameters of the model, $t_{a}, v_{\max }$ and $\tau$, provide a physically intuitive interpretation of the data. Analysis has revealed that pedestrians respond to shifting gap affordances primarily by timing their accelerations, rather than changing their walking speeds, as shown by the distributions of $t_{a}$. Moreover, shifts in $t_{a}$ have been observed in response to the speed of the gap and the size of the surrounding vehicles, even if the gap affordance remains the same, indicating that these environmental factors can change the visual perception of the gap. However, this trend disappeared when the initial distance was greater, suggesting that a greater distance from the road tends to offer a more accurate visual perception of the gap. It has also been observed that children's lower walking velocities indirectly shrank the affordances of the gaps and in certain situations failed to compensate.

Due to the simplicity of the equations, this methodology offers a versatile method to analyze pedestrian behavior. While the velocity equation does not necessarily need to follow a logistic function in particular, the high accuracy with which the equation fits the data and its ease of manipulation makes it an ideal tool for such an analysis.

It should be noted that the accuracy of affordance judgments in virtual environments has been questioned in previous studies [18]. In addition, it is not straightforward to measure walking speeds on treadmills, and a different method was proposed [19]. These factors should be considered when interpreting the results, but we expect them to affect neither qualitative results nor the efficacy of the model. Moreover, it would be desirable to include more general walking scenarios where the pedestrian is not constrained to walk in a straight line. This is left for future study.

\section{Conclusions}

We have utilized a newly developed model for crossing behavior to extract the control parameter used for adjusting to changing gaps, visualizing crossings within the gap affordance, and testing the bearing angle hypothesis. The model thus serves as a useful tool with which pedestrian behavior can be understood. It can also be used in the context of previous modeling frameworks and may further be developed to extract elements of crossing behavior which leads to collision.

Author Contributions: Conceptualization, M.C., J.W.K. and H.-C.C.; methodology, M.C. and J.W.K.; software, S.H.K.; visualization, S.H.K.; investigation, H.-C.C. and G.-J.C.; writing-original draft preparation, S.H.K.; writing-review and editing, J.W.K. and M.C.; All authors have read and agreed to the published version of the manuscript.

Funding: This research was funded by National Research Foundation of Korea through the Basic Science Research Program (Grant No. 2019R1F1A1046285) and by Korea Institute for Advancement of Technology and Ministry of Trade, Industry, and Energy (Grant No. 10044775).

Conflicts of Interest: The authors declare no conflict of interest.

\section{Appendix A. Analysis of Two-Step Crossings}

In order to model two-step crossings in which there are two acceleration events (Figure 3b), we extend the model in the following way: We first take the acceleration equation

$$
\ddot{y}=\frac{\dot{y}}{\tau}\left(1-\frac{\dot{y}}{v_{\max }}\right),
$$


which is equivalent to Equations 1 and 2 with appropriate initial conditions. In the two-step crossing, after acceleration (i.e., at time $t>t_{a}+2 \tau$ ), the pedestrian will decelerate at a point $y_{s}$ and stop until she/he accelerates again at time $t_{s}$. This behavior may be described by adding two terms in Equation (A1), which leads to

$$
\begin{aligned}
\ddot{y}= & \frac{\dot{y}}{\tau}\left(1-\frac{\dot{y}}{v_{\max }}\right)-r_{s} \dot{y} \exp \left[-\frac{\left(y-y_{s}\right)^{2}}{\sigma_{s}^{2}}\right] \theta\left(t_{s}-t\right) \\
& +v_{s} \delta\left(t-t_{s}\right),
\end{aligned}
$$

where the second and the third terms of the right-hand side represent repulsion and impulse force, respectively. The repulsion is centered at position $y_{s}$ with range $\sigma_{s} ; y_{s}$ may be interpreted as the point beyond which the pedestrian perceives to be unsafe, due to the incoming traffic. Accordingly, $y_{s}$ is the position of the flat region of the curve in Figure $3 b$, i.e., $y_{s}=-2.3 \mathrm{~m}$ in this example. The Heaviside step function $\theta\left(t_{s}-t\right)$ effectively "turns off" the repulsion force at time $t_{s}$, thus removing the potential for collision after the vehicle has passed. The parameter $r_{s}$ adjusts the overall strength of the repulsion. The impulse term is necessary for the model to undergo sharp acceleration from rest, so that the pedestrian starts to walk again at time $t_{s}$. In the example of Figure $3 \mathrm{~b}$, the time at which the subject begins the second acceleration is given by $t_{s}=2.0 \mathrm{~s}$. The magnitude $v_{s}$ of the impulse is a fraction of $v_{\max }$, and determines how quickly the model regains the maximum velocity.

We fit Equation (A2) to the data for the case $y_{0}=-3.5 \mathrm{~m}$ and $l_{g}=25 \mathrm{~m}$, yielding $y_{s}=-2.29 \pm 0.22 \mathrm{~m}$. This implies that participants who performed two-step crossings walked forward about $1.2 \mathrm{~m}$ before stopping, which amounts to about $1.5 \mathrm{~m}$ from the path of the vehicles. We also have the impulse magnitude $v_{s} / v_{\max }=0.67 \pm 0.22$ and time $t_{s}=2.41 \pm 0.26 \mathrm{~s}$, which corresponds to the time for the pedestrian to start walking again. This range includes the time (about $2.5 \mathrm{~s}$ ) at which the leading vehicle passes the interception point. Other parameters, repulsion strength $r_{s}$ and range $\sigma_{s}$, which suffer from large fluctuations due to the very limited sample number, are obtained as $r_{s}=520 \pm 480 \mathrm{~s}^{-1}$ and $\sigma_{s}=0.26 \pm 0.24 \mathrm{~m}$. However, the sample size of two-step crossings was insufficient to derive statistically meaningful results. A possible behavioral interpretation of this type of crossing is as exchanging the additional energy required for deceleration and acceleration in favor of more safety or security.

\section{References}

1. OECD. Road Infrastructure, Inclusive Development and Traffic Safety in Korea; OECD Publishing: Paris, France, 2016.

2. Hamed, M.M. Analysis of pedestrians' behavior at pedestrian crossings. Saf. Sci. 2001, 38, 63-82. [CrossRef]

3. Te Velde, A.F.; Van Der Kamp, J.; Savelsbergh, G.J.P. Five- to twelve-year-olds' control of movement velocity in a dynamic collision avoidance task Arenda. Br. J. Dev. Psychol. 2008, 26, 33-50. [CrossRef]

4. Azam, M.; Choi, G.-J.; Chung, H.-C. Perception of Affordance in Children and Adults While Crossing Road between Moving Vehicles Muhammad. Psychology 2017, 8, 1042-1052. [CrossRef]

5. Fajen, B.R.; Warren, W.H. Behavioral Dynamics of Steering, Obstacle Avoidance, and Route Selection Brett. J. Exp. Psychol. Hum. 2003, 29, 343-362. [CrossRef] [PubMed]

6. Chapman, S. Catching a Baseball. Am. J. Phys. 1968, 36, 868-870. [CrossRef]

7. Bastin, J.; Craig, C.; Montagne, G. Prospective strategies underlie the control of interceptive actions. Hum. Mov. Sci. 2006, 25, 718-732. [CrossRef] [PubMed]

8. Fajen, B.R.; Warren, W.H. Behavioral dynamics of intercepting a moving target. Exp. Brain Res. 2007, 180, 303-319. [CrossRef] [PubMed]

9. Gibson, J.J. The Ecological Approach to Visual Perception. In Visual Guidance of Intercepting a Moving Target on Foot; Houghton Mifflin Harcourt (HMH): Boston, MA, USA, 1979; p. 127.

10. Patla, A.E. Understanding the roles of vision in the control of human locomotion. Gait Posture 1997, 5, 54-69. [CrossRef] 
11. Warren, W.H.; Kay, B.A.; Zosh, W.D.; Duchon, A.P.; Sahuc, S. Optic flow is used to control human walking. Nat. Neurosci. 2001, 4, 213-216. [CrossRef] [PubMed]

12. Fajen, B.R.; Warren, W.H. Visual guidance of intercepting a moving target on foot. Perception 2004, 33, 689-715. [CrossRef] [PubMed]

13. Fajen, B.R. Guiding locomotion in complex, dynamic environments. Front. Behav. Neurosci. 2013, 7, 85. [CrossRef] [PubMed]

14. Plumert, J.M.; Kearney, J.K.; Cremer, J.F. Children's perception of gap affordances: Bicycling across traffic-filled intersections in an immersive virtual environment. Child Dev. 2004, 75, 1243-1253. [CrossRef] [PubMed]

15. Fajen, B.R.; Matthis, J.S. Direct perception of action-scaled affordances: The shrinking gap problem. J. Exp. Psychol. Hum. Percept. Perform 2011, 37, 1442-1457. [CrossRef] [PubMed]

16. Yannis, G.; Papadimitriou, E.; Theofilatos, A. Pedestrian gap acceptance for mid-block street crossing. Transp. Plan. Technol. 2013, 36, 450-462. [CrossRef]

17. Chihak, B.J.; Plumert, J.M.; Ziemer, C.J.; Kearney, J.K. Synchronizing self and object movement: How child and adult cyclists intercept moving gaps in a virtual environment. J. Exp. Psychol. Hum. Percept. Perform 2010, 36, 1535-1552. [CrossRef] [PubMed]

18. Creem-Regehr Sarah, H.; Gill, D.M.; Pointon, G.D.; Bodenheimer, B.; Stefanucci, J. Mind the gap: Gap affordance judgments of children, teens, and adults in an immersive virtual environment. Front. Robot. AI 2019, 6, 96. [CrossRef]

19. Yoon, J.; Park, H.S.; Damiano, D.L. A novel walking speed estimation scheme and its application to treadmill control for gait rehabilitation. J. Neuroeng. Rehabil. 2012, 9, 62. [CrossRef] [PubMed]

(C) 2020 by the authors. Licensee MDPI, Basel, Switzerland. This article is an open access article distributed under the terms and conditions of the Creative Commons Attribution (CC BY) license (http://creativecommons.org/licenses/by/4.0/). 